\title{
Enhancing engagement in medical leadership through structured quality improvement teaching
}

\author{
Authors: Lahiru Satharasinghe, ${ }^{A}$ Rachael Slater ${ }^{A}$ and Simon Conroy ${ }^{B}$
}

\section{Aims}

To evaluate the impact of an integrated clinical quality improvement module within undergraduate medical and nursing training on leadership skills development using the domains of the Medical Leadership Competency Framework (MLCF).

\section{Methods}

The quality improvement module initially comprised formal methodology lectures and supervised projects in an acute hospital setting. The 10-week module was interdisciplinary; mixed small groups of undergraduate third-year medical and nursing students worked alongside clinical fellows. Following completion of one Plan, Do, Study, Act cycle, the results were analysed and recommendations to improve clinical practice were relayed through video and oral presentation to senior clinicians, senior managers, patient safety group leads, patient forum members and local charities.

Post-course evaluation of the module was undertaken through an anonymised questionnaire. This was administered using SurveyMonkey to the 23 module participants. Questions were structured to reflect the domains of the MLCF and Kirkpatrick's Four-Level Training Evaluation Model. The survey included free text and Likert questions. Responses were collected and analysed for evidence of change in behaviour, attitude and learning.

\section{Results}

The post-course questionnaire response rate was $65 \%$, with the 15 respondents consisting of 13 medical students and two nursing students. The responses around personal qualities showed that students could reflect and identify their individual strengths, such as organisational and team-working skills, while also noting their development of weaker areas such as presentation skills and confidence. They demonstrated improved assertiveness and effective communication with others. $92 \%$ of respondents agreed that the module improved their knowledge of hospital working and students gained a better understanding of how staff groups in hospital make decisions. Overall, $79 \%$ of respondents felt more confident in improving patient safety within the hospital setting and could identify barriers to change. There was more difficulty in achieving the MLCF domain focused on setting direction, as many did not feel that they could apply the knowledge they gained or use it to influence other people.

\section{Conclusions}

This questionnaire has identified that many aspects of leadership development have been taken from this quality improvement student-selected module. There were MLCF domain questions around practical application that returned negative responses; however, this may be due to the infrequent opportunities to put leadership in practice within a preclinical undergraduate curriculum. This survey supports the use of practical quality improvement in an interdisciplinary setting to develop leadership abilities within a short timescale.

\section{Conflict of interest statement}

None declared.

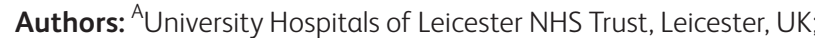
${ }^{B}$ University of Leicester, Leicester, UK 